\title{
Clinical significance of plasma globotriaosylsphingosine levels in Chinese patients with Fabry disease
}

\author{
YAN OUYANG $^{1 *}$, BING CHEN $^{2 *}$, XIAOXIA PAN ${ }^{1}$, ZHAOHUI WANG $^{1}$, HONG REN $^{1}$, \\ YAOWEN XU ${ }^{1}$, LIYAN NI $^{1}$, XIALIAN YU ${ }^{1}$, LI YANG $^{1}$ and NAN CHEN ${ }^{1}$ \\ ${ }^{1}$ Department of Nephrology, Institute of Nephrology, Ruijin Hospital, The Medical School of Shanghai Jiao Tong University; \\ ${ }^{2}$ Department of Pharmacy, Ruijin Hospital, The Medical School of Shanghai Jiao Tong University, \\ Shanghai 200025, P.R. China
}

Received October 21, 2016; Accepted December 6, 2017

DOI: $10.3892 /$ etm.2018.5889

\begin{abstract}
Although plasma globotriaosylsphingosine (lyso-Gb3) is a promising biomarker of Fabry disease (FD), few studies have assessed the impact of lyso-Gb3 in patients with FD. A total of 38 patients diagnosed with FD at Ruijin Hospital between January 2012 and December 2014 were recruited in the current study. An additional 120 unrelated healthy individuals were selected as healthy controls. A simplified liquid chromatography-tandem mass spectrometry (LC-MS/MS) assay was performed to determine lyso-Gb3 levels in plasma. Protein precipitation and glycolipid extraction were conducted using acetone/methanol. Clinical performance, including diagnostic value and disease surveillance, were compared between plasma lyso-Gb3 levels and $\alpha$-galactosidase A ( $\alpha$-gal A) enzyme activity. The overall coefficient of variation values between inter- and intra-days varied between 2.8 and $18.9 \%$ and linearity correlation coefficients were $\geq 0.99$ for all assays. Therefore, the effectiveness of the LC-MS/MS method was validated. Furthermore, a cut-off value of $0.81 \mathrm{ng} / \mathrm{ml}$ plasma lyso-Gb3 was able to separate patients with FD from healthy individuals. The sensitivity of this cut-off was $94.7 \%$ and the specificity was $100 \%$. Compared with $\alpha$-gal A enzyme activity, the diagnostic rate of patients assessed using plasma lyso-Gb3 levels was similar; however, there was a tighter correlation between plasma lyso-Gb3 levels and the mainz severity score index score in male patients $(r=0.711$ vs. $r=-0.687)$. The sensitivity of plasma lyso-Gb3 in diagnosing female patients with FD was higher than $\alpha$-gal A enzyme activity (82.4 vs. 23.5\%).
\end{abstract}

Correspondence to: Dr Nan Chen, Department of Nephrology, Institute of Nephrology, Ruijin Hospital, The Medical School of Shanghai Jiao Tong University School of Medicine, 197 Ruijin ER Road, Shanghai 200025, P.R. China

E-mail: cnrj100@126.com

*Contributed equally

Key words: Fabry disease, plasma globotriaosylsphingosine, liquid chromatography-tandem mass spectrometry, enzyme activity, clinical application
To the best of our knowledge, the present study is the first to report the effectiveness of plasma lyso-Gb3 levels in diagnosing Chinese patients with FD. Using $\alpha$-gal A activity as a reference, the results of current study indicated that plasma lyso-Gb3 levels are more useful at diagnosing female patients with FD. Furthermore, plasma lyso-Gb3 levels are more suitable at determining overall disease severity in male patients.

\section{Introduction}

Fabry disease (FD) is an X-linked genetic disease resulting from the deficient enzyme activity of $\alpha$-galactosidase $\mathrm{A}$ [ $\alpha$-gal A; Online Mendelian Inheritance in Man of the galactisidase (GLA) gene, 301500; Enzyme Commission number of $\alpha$-gal A, 3.2.1.22], which is encoded by the GLA gene (Xq22) (1). This enzymatic defect leads to the accumulation of glycolipid, which consists primarily of globotriaosylceramide (Gb3), in tissues, organs and biological fluids (2). Over time, a gradual deterioration in organ function occurs, resulting in corresponding renal, cardiac and vascular complications (3). The early diagnosis and treatment of FD is vital to prevent irreversible damage to vital organs; however, FD is often misdiagnosed due to its heterogeneous clinical manifestations and inefficient diagnostic methods, such as enzyme assays (3-5).

In males, the diagnosis of FD is usually based on the dramatically decreased activity of $\alpha$-gal A (6). However, enzyme assays are usually inconclusive at diagnosing FD in females, as $\alpha$-gal A activity usually ranges between relatively low to normal levels in females with FD (7). The association between Gb3 levels and $\alpha$-gal A activity is weak and it has therefore been suggested is that Gb3 is not ideal as a primary diagnostic marker of FD (8-11). Although genotyping is a powerful diagnostic tool, the rate of mutation detection remains at $\sim 10 \%(12,13)$; therefore it is difficult to identify novel GLA variants, as FD lacks defining clinical characteristics (14). To avoid the misdiagnosis of individuals with an unknown genetic composition, non-invasive methods, including imaging examinations, may be used. However, these are often unable to exclude FD in uncertain cases $(15,16)$. These patients are classified as uncertain cases as they exhibit uncharacteristic FD manifestations and lack of a family history of the disease; such patients only exhibited solitary unexplained cardiac hypertrophy or 
stroke. Biopsy results from organs affected by FD, such as the heart or kidneys, reveal the presence of characteristic lamellar inclusion bodies following electron microscopy assessment, which also confirms FD diagnosis $(17,18)$. However, biopsies are invasive and an unfeasible method of diagnosing all patients with FD. Therefore, a novel diagnostic biomarker of FD is urgently required.

In 2008, globotriaosylsphingosine (lyso-Gb3) was introduced as a promising novel biomarker of FD, which has better diagnostic sensitivity than $\alpha$-gal A (19). Lyso-Gb3 is a cationic amphiphilic compound containing a polar sugar group and is hydrophilic. It is also a $\mathrm{Gb} 3$ metabolite and contains a free sphingosine amino group. As lyso-Gb3 is able to freely access cells, it is more easily detectable than Gb3 (20). Certain methods used to measure lyso-Gb3, such as high-performance liquid chromatography (HPLC), o-phthaldialdehyde (OPA)-derivatization or fluorescence detection (19-21), did not exhibit high sensitivity to lyso-Gb3. Previous studies have suggested that liquid chromatography-tandem mass spectrometry (LC-MS/MS) may be a more suitable technique of measuring lyso-Gb3 (22-25). Lyso-Gb3-related analogues have been identified as novel biomarkers for FD (26-29). However, levels of these analogues are much lower than lyso-Gb3 in human plasma and are undetectable in some patients with FD and healthy subjects. Therefore, the current study focused on measuring lyso-Gb3 levels in human plasma.

To the best of our knowledge, the current study is the first to measure plasma levels of lyso-Gb3 in Chinese patients with FD. A simplified LC-MS/MS assay was used to measure lyso-Gb3 levels. An internal standard (IS) used to analyze sphingolipid metabolism is easy to obtain and used to quantify lyso-Gb3 levels. In addition, the clinical significance of $\alpha$-gal A activity and lyso-Gb3 were determined and compared to determine which would more suitable for use in patients with FD.

\section{Patients and methods}

Patients and samples. Preliminary diagnoses of FD were based on the presence of a myeloid body in histological studies and/or decreased $\alpha$-gal A enzyme activity; patients with FD exhibit $\alpha$-gal A enzyme activity $\leq 37.0 \mathrm{nmol} / \mathrm{ml} / \mathrm{h} / \mathrm{mg}$ (30-32). FD diagnosis was confirmed by genetic sequencing, as previously described (32). Between January 2012 and December 2014, 38 patients with FD (17 females and 21 males, 1:1.24; age, $34.7 \pm 16.0$ years) and 120 healthy volunteers (60 females and 60 males, 1:1; age, $40.8 \pm 10.9$ years) were enrolled at Ruijin Hospital, The Medicine School of Shanghai Jiao Tong University (Shanghai, China). The present study was approved by the Ethics Committee of Ruijin Hospital and all participants provided their informed consent prior to participation in the current study. Written informed consent was obtained from the parents or guardians of children enrolled. All EDTA-anti-coagulated blood samples from patients with FD were collected prior to enzyme replacement therapy (ERT).

Demographic and clinical variables. For all patients with FD, demographic data, including age, sex, blood pressure and body mass index, as well as their clinical symptoms were recorded. Estimated glomerular filtration rate (eGFR) was estimated using the abbreviated chronic kidney disease epidemiology collaboration equation (33) in adults and the Schwartz formula (34) in children. End-stage renal disease was defined as an eGFR $<15 \mathrm{ml} / \mathrm{min}$ or the requirement of kidney transplant or dialysis. Abnormal brain magnetic resonance imaging (MRI) results were indicated by the presence of white matter lesions and lacunar infarctions. Left ventricular hypertrophy was identified by echocardiography. Cornea verticillata and high frequency sensorineural hearing loss were confirmed by the slit-lamp examination and pure tone audiogram, respectively. The presence of acroparesthesia was determining by assessing the medical history of patients and identifying the presence of neuropathic pain in the palms and soles. Physical examination and history taking were used to detect angiokeratoma corporis diffusum and abnormal sweating. Based on these data, the Mainz severity score index (MSSI) was scored to evaluate overall disease severity (35). Patients included in the current study either exhibited classical FD phenotypes or atypical FD phenotypes, which were defined by the diagnostic criteria described in a previous study by our group (32).

Reagents. For LC-MS/MS, lyso-Gb3 was purchased as standard (Matreya LLC, State College, PA, USA) whereas dimethyl psychosine was selected as the IS (Avanti Polar Lipids, Alabastar, AL, USA). Pure water (AppliChem GmbH, Darmstadt, Germany), acetonitrile and methanol (Thermo Fisher Scientific, Inc., Waltham, MA, USA), formic acid and ammonium formate (Sigma-Aldrich; Merck KGaA, Darmstadt, Germany) and all other chemicals were HPLC grade. To detect the $\alpha$-gal A enzyme activity, 4-methylumbelliferyl- $\alpha$-D-galactopyranoside (4MU-gal), 4MU and $\mathrm{N}$-acetyl-D-galactosamine (galNAc) were purchased from Sigma-Aldrich; Merck KGaA.

Preparation of EDTA-plasma for lyso-Gb3. A total of $3 \mathrm{ml}$ whole blood was drawn from the elbow vein of patients with FD and centrifuged at $1,500 \mathrm{xg}$ for $10 \mathrm{~min}$ at $4^{\circ} \mathrm{C}$. Plasma was separated and stored at $-20^{\circ} \mathrm{C}$ until use. The neat lyso-Gb3 standard was dissolved as recommended by the supplier and diluted with methanol to a working solution concentration of $10 \mu \mathrm{g} / \mathrm{ml}$. Aliquots of $100 \mu \mathrm{l}$ standard were dried and stored at $-20^{\circ} \mathrm{C}$ for later use. For each analysis, a blank plasma pools from healthy controls were used for preparing the calibration standards and quality control (QC) samples. After adding appropriate volumes of the working solution to blank plasma matrix, the final calibration standards concentrations of lysoGb3 were $200,100,50,25,12.5,6.25,3.13,1.56,0.78$ and $0 \mathrm{ng} / \mathrm{ml}$. Final QC samples concentrations of lysoGb3 were 4, 20 and $160 \mathrm{ng} / \mathrm{ml}$. Glycolipids were extracted from $50 \mathrm{ml}$ plasma samples according to simultaneous protein precipitation (36), which was performed by adding a mixture of acetone and methanol $(1 \mathrm{ml}, 1: 1)$ to a plasma sample and centrifuging the mixture at $14,000 \mathrm{x}$ g for $10 \mathrm{~min}$ at $4^{\circ} \mathrm{C}$ to remove the sediment. The supernatant was collected and evaporated for LC-MS/MS analysis without the requirement of further sample preparation, such as Solid-Phase Extraction. For analysis, samples were re-dissolved in $100 \mu 1$ pure methanol with subsequent sonication at $40 \mathrm{kHz}$ for $10 \mathrm{~min}$, 
A

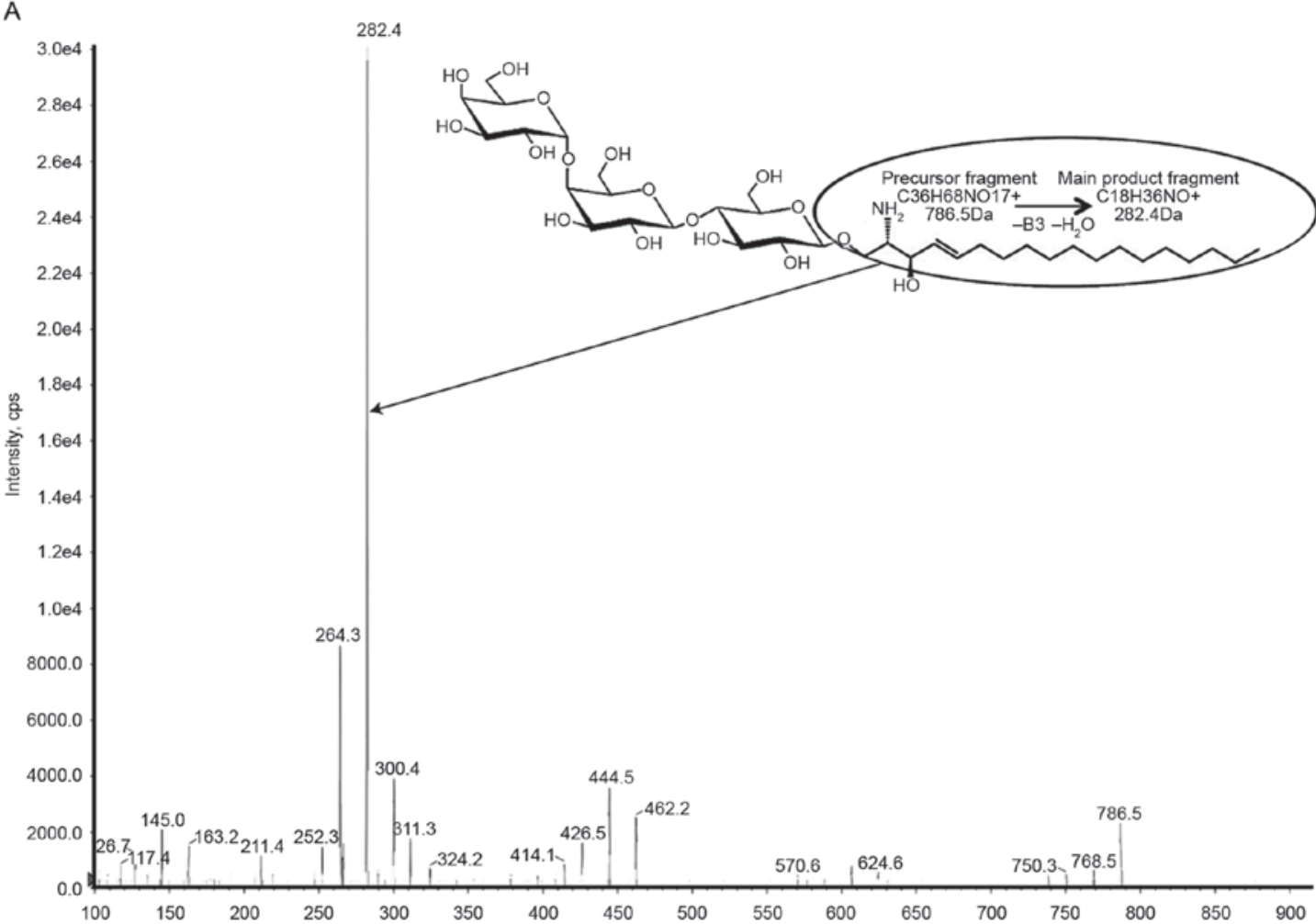

B

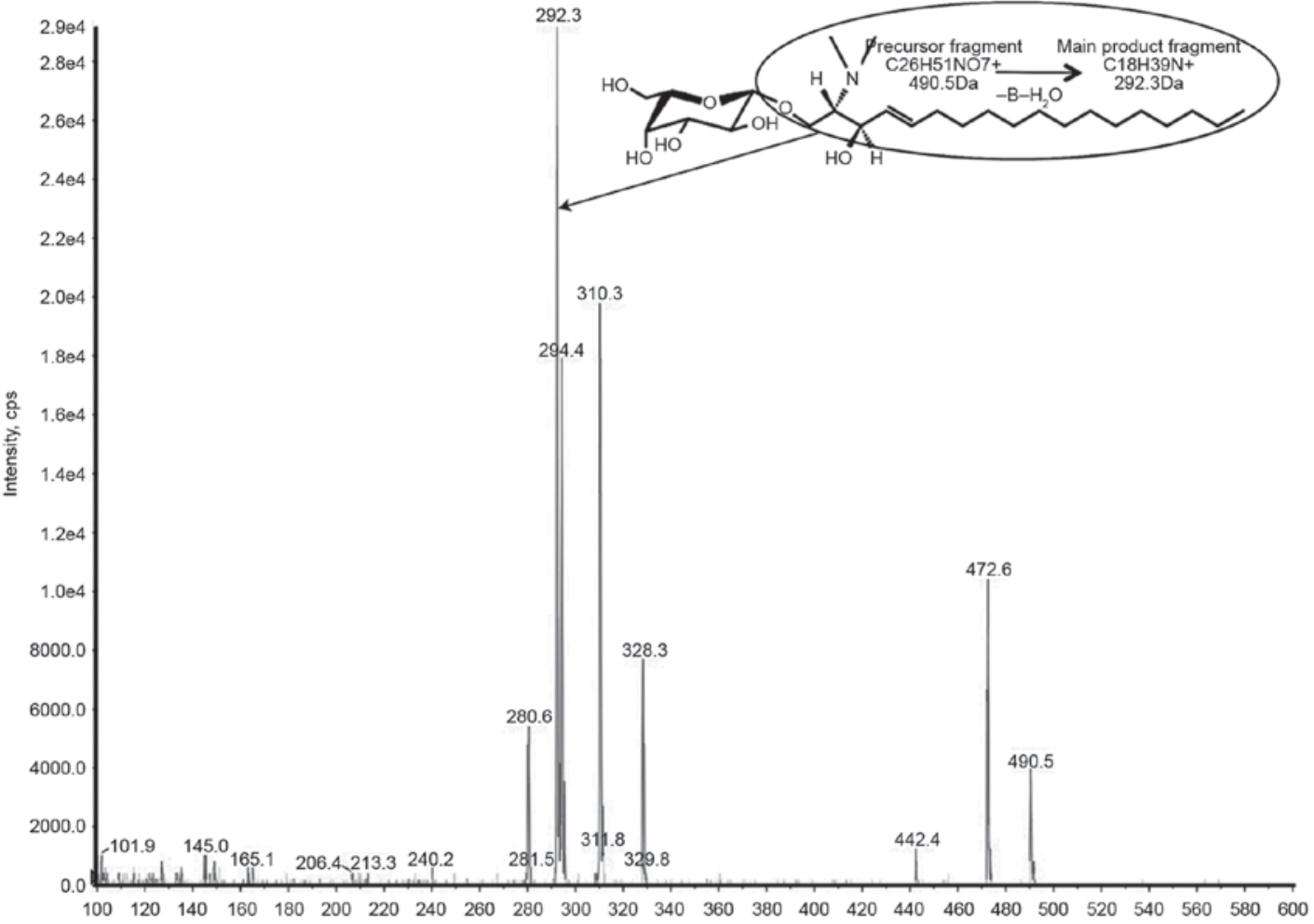

$\mathrm{m} / \mathrm{z}$

Figure 1. The product ion spectra of lyso-Gb3 and dimethyl psychosine are presented. (A) Fragmentation spectrum of lyso-Gb3. The predominant product ion $(\mathrm{m} / \mathrm{z} 282.4)$ generated by the collision-induced fragmentation of lyso-Gb3 $(\mathrm{m} / \mathrm{z} 786.5)$ is indicated. (B) Fragmentation spectrum of dimethyl psychosine. For this internal standard, electrospray detection as $[\mathrm{M}+\mathrm{H}]+(\mathrm{m} / \mathrm{z} 490.5)$ is straightforward due to its basic amino group. Collision induced dissociation results in the formation of a prominent fragment (m/z 292.3) resembling the sphingosine moiety, which gives the possibility of monitoring the internal standard with high specificity via multiple-reaction monitoring transition 490.5>292.3. Lyso-Gb3, globotriaosylsphingosine.

followed by vortexing at 1,200 rpm for 3 min and centrifugation at $14,000 \mathrm{x}$ g for $10 \mathrm{~min}$ at $4^{\circ} \mathrm{C}$. The supernatant was transferred into glass microvials and $5 \mu \mathrm{l}$ was injected into the LC-MS/MS system. 
Table I. Clinical baseline characteristics of male and female patients with Fabry disease.

\begin{tabular}{|c|c|c|c|}
\hline Parameter & Male $(n=21)$ & Female $(\mathrm{n}=17)$ & P-value \\
\hline Enzyme activity $(\mathrm{nmol} / \mathrm{ml} / \mathrm{h} / \mathrm{mg})$ & $0.3(0.0-23.2)$ & $64.4(14.8-152.4)$ & $<0.001$ \\
\hline Lyso-Gb3 (ng/ml) & $14.5(0.9-113.4)$ & $2.8(0.7-6.0)$ & $<0.001$ \\
\hline $\operatorname{BMI}\left(\mathrm{kg} / \mathrm{m}^{2}\right)$ & $23.1 \pm 7.1$ & $22.0 \pm 6.9$ & 0.647 \\
\hline Onset age (year) & $13(1-51)$ & $33(5-54)$ & 0.114 \\
\hline Age at diagnosis (year) & $31.1 \pm 14.6$ & $39.2 \pm 16.8$ & 0.122 \\
\hline $\mathrm{SBP}(\mathrm{mmHg})$ & $132.2 \pm 23.7$ & $130.1 \pm 13.7$ & 0.765 \\
\hline $\mathrm{DBP}(\mathrm{mmHg})$ & $81.8 \pm 15.7$ & $77.6 \pm 9.1$ & 0.378 \\
\hline $\mathrm{PP}(\mathrm{mmHg})$ & $50.4 \pm 15.6$ & $52.5 \pm 11.2$ & 0.674 \\
\hline MAP (mmHg) & $98.6 \pm 17.3$ & $95.1 \pm 9.5$ & 0.461 \\
\hline Angiokeratoma, \% (N) & $20.0(4 / 20)$ & $7.1(1 / 14)$ & 0.298 \\
\hline Abnormal sweating, \% (N) & $35.0(7 / 20)$ & $7.1(1 / 14)$ & 0.059 \\
\hline Cornea verticillata, \% (N) & $70.0(14 / 20)$ & $50.0(7 / 14)$ & 0.238 \\
\hline Hearing loss, \% (N) & $37.5(6 / 16)$ & $16.7(2 / 12)$ & 0.227 \\
\hline Acroparaesthesia, \% (N) & $50.0(10 / 20)$ & $50.0(7 / 14)$ & 0.999 \\
\hline Brain MRI, \% (N) & $21.1(4 / 19)$ & $38.5(5 / 13)$ & 0.282 \\
\hline $\mathrm{LVH}, \%(\mathrm{~N})$ & $21.1(4 / 19)$ & $0.0(0 / 14)$ & 0.067 \\
\hline ESRD, \% (N) & $14.3(3 / 21)$ & $0.0(0 / 17)$ & 0.104 \\
\hline Classical phenotype, \% (N) & $47.6(10 / 21)$ & $35.3(6 / 17)$ & 0.521 \\
\hline MSSI score & $19.6 \pm 10.0$ & $7.6 \pm 5.1$ & $0.003^{\mathrm{a}}$ \\
\hline
\end{tabular}

${ }^{\mathrm{a}} \mathrm{P}<0.05$. Continuous variables are presented as mean \pm standard deviation or median (minimum-maximum). As the MSSI score required comprehensive data of all the examinations, 15 patients ( 7 males and 8 females) lacking $\geq 1$ examination result were excluded from the MSSI scoring system. Lyso-Gb3, globotriaosylsphingosine; BMI, body mass index; SBP, systolic blood pressure; DBP, diastolic blood pressure; PP, Pulse pressure; MAP, mean arterial pressure; MRI, magnetic resonance imaging; LVH, left ventricular hypertrophy; ESRD, end-stage renal disease; MSSI, mainz severity score index.

LC-MS/MS quantification of lyso-Gb3. Quantitative LC-MS/MS analysis was performed on an AB SCIEX API 4000 triple quadrupole mass spectrometer (AB Sciex LLC, Framingham, MA, USA) operating in positive mode. An XDB-C18 column ( $2.1 \times 100 \mathrm{~mm}, 3.5 \mu \mathrm{m}$ particles) was used in combination with a VanGuard pre-column of the same material (Agilent Technologies, Inc., Santa Clara, CA, USA). The mobile phases consisted of (A) water and (B) acetonitrile/methyl alcohol $(15 / 85=\mathrm{v} / \mathrm{v})$. Each mobile phase contained $0.1 \%$ formic acid and $2 \mathrm{mM}$ ammonium formate. The two mobile phases formed the following gradient: $0-0.25(50 \% \mathrm{~B}) \mathrm{min}$, 0.25-2.0 $\mathrm{min}(50-99 \%, \mathrm{~B}), 2.0-4.5 \mathrm{~min}(99 \%, \mathrm{~B}), 4.5-4.75 \mathrm{~min}$ $(99-50 \% \mathrm{~B})$ and $4.75-8.5 \mathrm{~min}(50 \%, \mathrm{~B})$. The MS conditions were as follows: ESI mode was positive, ion spray voltage was $5.5 \mathrm{KV}$, nebulizer gas and auxiliary gas were 60 psi, curtain gas was $25 \mathrm{psi}$, the desolvation temperature was $600^{\circ} \mathrm{C}$ and the flow rate is $0.3 \mathrm{ml} / \mathrm{min}$. The following multiple-reaction monitoring (MRM) transitions were monitored, with a dwell time of $100 \mathrm{~ms}$ : 786.5>282.4 (lyso-Gb3) and 490.5>292.3 (IS). Collision energy was $48.6 \mathrm{~V}$ and $38 \mathrm{~V}$ in the MRM traces of lyso-Gb3 and IS, respectively (Fig. 1).

Measurement of $\alpha$-gal A activity. $\alpha$-gal A activity in leukocytes was detected using the fluorimetric method, as described by Desnick et al (31). Briefly, $4 \mathrm{ml}$ whole blood was added to 50-ml conical tubes. Leukocyte pellets were isolated and resuspended in $100 \mu \mathrm{l}$ sodium phosphate buffer. Following 10 cycles of freezing/thawing, leukocyte lysates were centrifuged at $2,000 \mathrm{x} \mathrm{g}$ for $10 \mathrm{~min}$ at $4^{\circ} \mathrm{C}$. Supernatants were stored at $-20^{\circ} \mathrm{C}$ to allow assaying at a later period. The mixture of $4 \mathrm{MU}-\mathrm{gal}$ plus galNAc solution was added to the leukocyte lysate and incubated at $37^{\circ} \mathrm{C}$ for $30 \mathrm{~min}$. Reactions were quenched using 0.1 M Na-Glycine buffer (pH 10.7). Fluorescence readings were performed using a Turner Fluorometer (Turner Designs, San Jose, CA, USA) using the following wavelengths: Excitation at $360 \mathrm{~nm}$ and emission at $450 \mathrm{~nm}$. Protein values were determined using a standard BCA assay and were used to calculate the specific enzyme activity of the lysates. Normal $\alpha$-gal A activity was defined as $>37.0 \mathrm{nmol} / \mathrm{ml} / \mathrm{h} / \mathrm{mg}(32)$.

Statistical analysis. The distributions of quantitative variables were assessed for normality. Results are presented as mean \pm standard deviation or median (range). All analyses were performed using IBM SPSS Statistics, ver. 22 (IBM Corp, Armonk, NY, USA). A Mann-Whitney U-test was used to assess differences between two non-parametric variables, and a t-test was used to compare two normally distributed variables. To analyze the relationship between two variables, the correlation coefficient was calculated using Spearman's correlation coefficient. A receiver operating characteristic (ROC) curve was used to define the pathological cutoff point of lyso-Gb3 and compare the diagnostic value between it and enzyme activity. $\mathrm{P}<0.05$ was considered to indicate a statistically significant difference. 
A

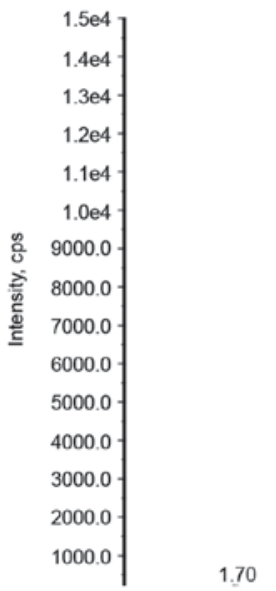

B

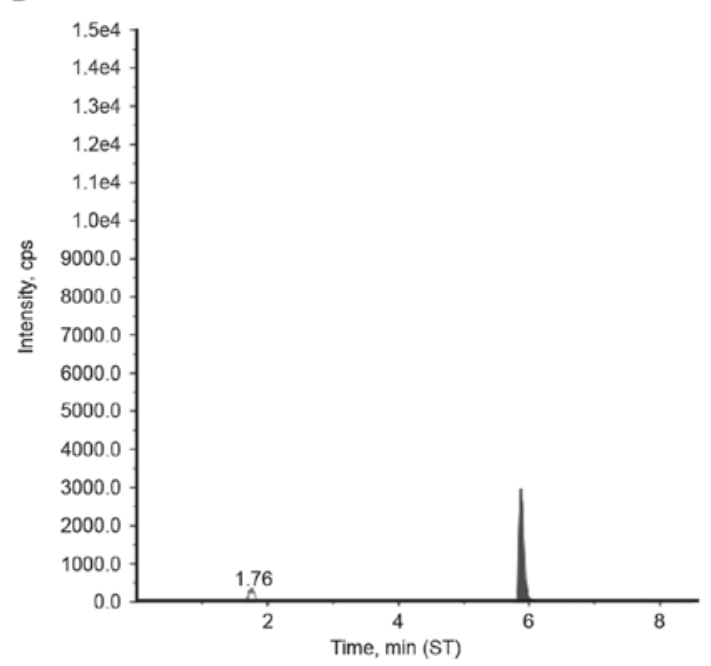

C

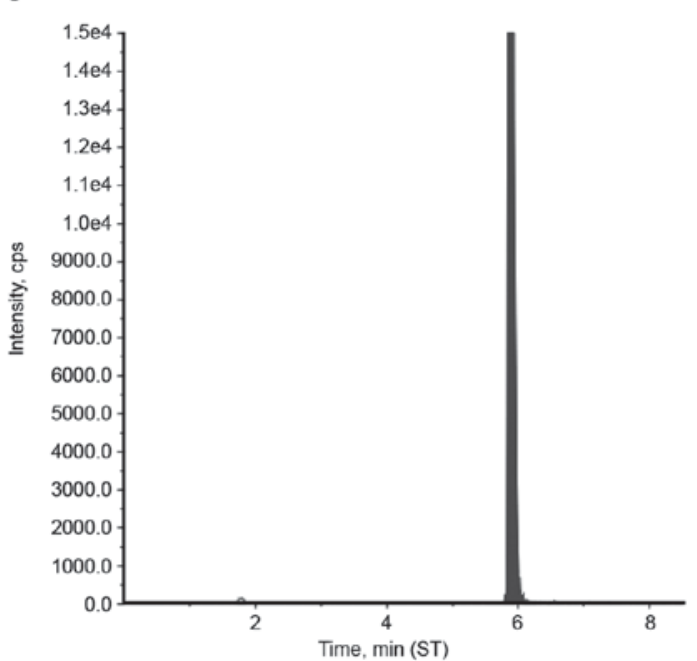

Normal

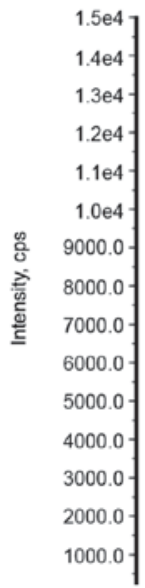

Female

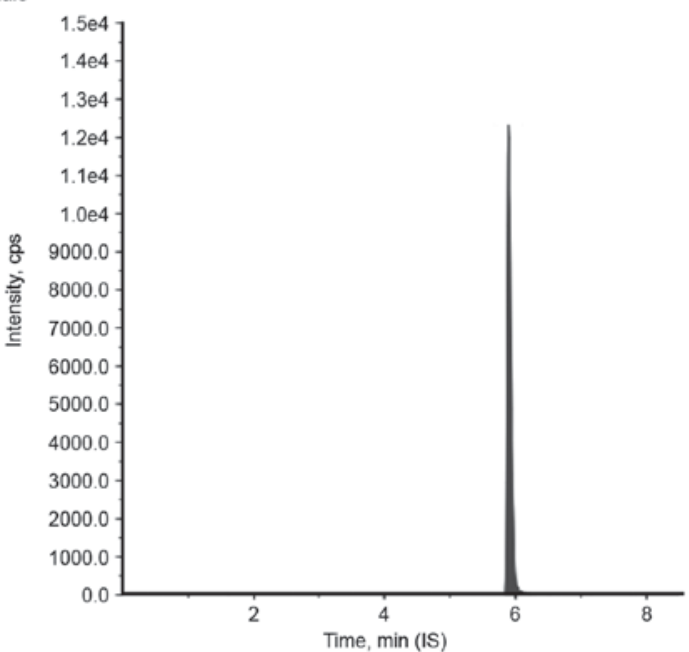

Male

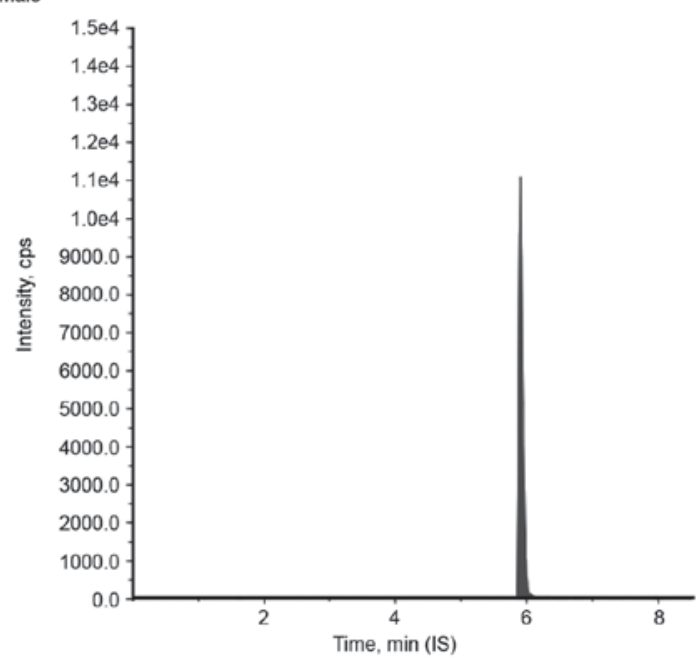

Figure 2. Verified specificity of lyso-Gb3 in EDTA plasma. (A) A normal volunteer, (B) an untreated female and (C) an untreated male with FD. For validation, lyso-Gb3 was used as ST while dimethyl psychosine was applied as IS. The retention time of ST (left peak) is less than that of the IS (right peak). FD, Fabry disease; lyso-Gb3, globotriaosylsphingosine; ST, standard; IS, internal standard; cps, counts per second.

\section{Results}

Demographic characteristics. The demographic variables of the 38 patients with FD prior to ERT are presented in Table I. Compared with female patients, males exhibited an earlier age of onset (13 vs. 33 years). Cornea verticillata 
Table II. Imprecision, inaccuracy and recovery results for globotriaosylsphingosine quantification.

\begin{tabular}{lccc}
\hline Quality control samples & Low & Medium & High \\
\hline Mean value \pm SD, g/l & $4.1 \pm 0.3$ & $20.1 \pm 1.9$ & $165.8 \pm 12.7$ \\
CV\% within-run (batch) & 9.9 & 7.2 & 4.6 \\
CV\% estimate of between-day & 18.9 & 14.6 & 2.8 \\
CV\% estimate of between-run & $0^{\mathrm{a}}$ & $0^{\mathrm{a}}$ & $0^{\mathrm{a}}$ \\
CV\% estimate of within-run & 12.0 & 8.9 & 10.8 \\
Recovery \%, mean \pm SD & $86.3 \pm 5.9$ & $92.0 \pm 3.0$ & $97.0 \pm 3.6$ \\
Recovery max, \% & 93 & 95 & 101 \\
Recovery min, \% & 82 & 89 & 94
\end{tabular}

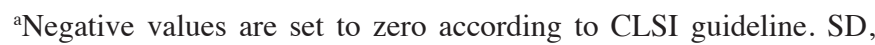
standard deviation; $\mathrm{CV}$, coefficient of variation.

was the presenting symptom most frequently reported by males and females (70\% of males and $50 \%$ of females reported experiencing this symptom). Males with FD were more likely to report a clinical event, including end-stage renal disease and life threatening cardiovascular or cerebrovascular complications, compared with females. Male patients exhibited significantly higher levels of lyso-Gb3 accumulation and lower enzyme activity (each, $\mathrm{P}<0.001$ ) and also presented with significantly higher MSSI scores than females $(\mathrm{P}<0.05)$.

Assay validation of the quantitation of plasma lyso-Gb3. An individual from each group was selected as a typical representative; the chromatography of LC-MS/MS results revealed that a male patient displayed with a lyso-Gb3 level of $106.5,6.04 \mathrm{ng} / \mathrm{ml}$ for a female patient and $0.61 \mathrm{ng} / \mathrm{ml}$ for a healthy male control (Fig. 2). Diagnostic assay validation was performed according to the criteria of the Clinical and Laboratory Standards Institute $(37,38)$. Linearity was tested by a dilution series of blank plasma pools analyzed in three separate runs. In this way, the limit of detection representing the assay background $(0.22 \pm 0.04 \mathrm{ng} / \mathrm{ml})$ was calculated from all subsequent sample dilutions that did not lead to further signal reduction. Recoveries of 86.3-97.0\% were measured in QC samples prior to and following extraction (Table II). Intra-assay variation, determined by analyzing a pooled sample in one batch, was 4.6-9.9\%. Inter-assay variation, determined by analyzing individually prepared samples in different runs over different days, was $2.8-18.9 \%$. Methanolic stock solutions of the standard and IS were stable for $\geq 2$ months at $-20^{\circ} \mathrm{C}$. Samples and controls were also frozen at $-20^{\circ} \mathrm{C}$ and did not exhibit a significant decrease following 2 months storage. There were no noticeable influences on quantitative results $\leq 3$ freeze-thaw cycles. These results reveal that using the LC-MS/MS method to quantify plasma lyso-Gb3 levels is accurate, reproducible and highly sensitive.

Diagnostic performance of plasma lyso-Gb3 and $\alpha$-gal A enzyme activity. No significant difference in $\alpha$-gal A enzyme activity was observed between male and female
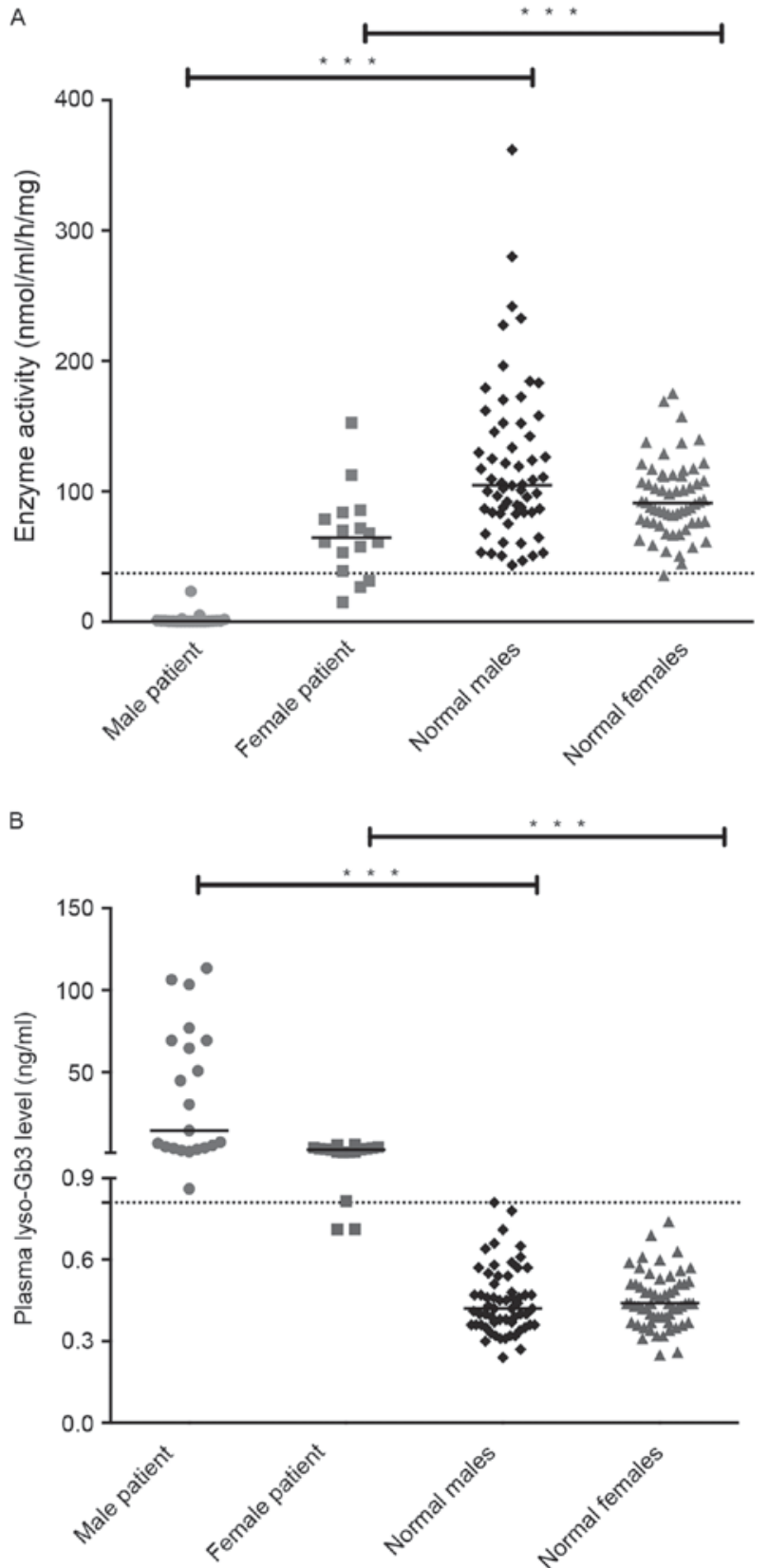

Figure 3. Differences in plasma lyso-Gb3 levels and enzyme activity were detected in males and females with Fabry disease. (A) Enzyme activity and (B) lyso-Gb3 levels in the plasma of male patients $(n=21)$, female patients $(n=17)$, healthy males $(n=60)$ and healthy females $(n=60)$. Lines represent the median value in each group. ${ }^{* * *} \mathrm{P}<0.001$. Lyso-Gb3, globotriaosylsphingosine.

controls (male, median $104.7 \mathrm{nmol} / \mathrm{ml} / \mathrm{h} / \mathrm{mg}$; range, $43.2-361.8 \mathrm{nmol} / \mathrm{ml} / \mathrm{h} / \mathrm{mg}$; female, median $90.8 \mathrm{nmol} / \mathrm{ml} / \mathrm{h} / \mathrm{mg}$; range, $35.5-175.1 \mathrm{nmol} / \mathrm{ml} / \mathrm{h} / \mathrm{mg}$; Fig. 3A). By contrast, enzyme activities in leukocytes of male patients with FD (median, $0.3 \mathrm{nmol} / \mathrm{ml} / \mathrm{h} / \mathrm{mg}$; range, $0.0-23.2 \mathrm{nmol} / \mathrm{ml} / \mathrm{h} / \mathrm{mg}$ ) were significantly lower than that of female patients with FD (median, $64.4 \mathrm{nmol} / \mathrm{ml} / \mathrm{h} / \mathrm{mg}$; range $14.8-152.4 \mathrm{nmol} / \mathrm{ml} / \mathrm{h} / \mathrm{mg}$ ) $(\mathrm{P}<0.001$; Table I). The significant differences were also observed between male patients and male healthy controls, and between female patients and female controls.

To verify the results of this assay and determine the normal range of lyso-Gb3, 120 healthy controls were included in the present study. No significant differences in lyso-Gb3 
Table III. Diagnostic sensitivity of LysoGb3 and enzyme activity.

\begin{tabular}{lccc}
\hline & \multicolumn{2}{c}{ Patients, N (\%) } & \\
\cline { 2 - 3 } $\begin{array}{l}\text { Diagnostic } \\
\text { biomarkers }\end{array}$ & $\begin{array}{c}\text { Male } \\
(\mathrm{N}=21)(\%)\end{array}$ & $\begin{array}{c}\text { Female } \\
(\mathrm{N}=17)(\%)\end{array}$ & P-value \\
\hline LysoGb3 & & & 0.193 \\
$>0.81 \mathrm{ng} / \mathrm{ml}$ & 100 & 82.4 & \\
$\leq 0.81 \mathrm{ng} / \mathrm{ml}$ & 0 & 17.6 & \\
$\begin{array}{l}\text { Enzyme activity } \\
>37 \mathrm{nmol} / \mathrm{ml} / \mathrm{h} / \mathrm{mg}\end{array}$ & 0 & 76.5 & $<0.001$ \\
$\leq 37 \mathrm{nmol} / \mathrm{ml} / \mathrm{h} / \mathrm{mg}$ & 100 & 23.5 & \\
\hline
\end{tabular}

The pathological cut-off for lyso-Gb3 level was $>0.81 \mathrm{ng} / \mathrm{ml}$. The pathological cut-off for $\alpha$-galactosidase A enzyme activity in leukocytes was $\leq 37 \mathrm{nmol} / \mathrm{ml} / \mathrm{h} / \mathrm{mg}$, respectively. The diagnostic sensitivities of two biomarkers were compared between male and female patients. Lyso-Gb3, globotriaosylsphingosine.

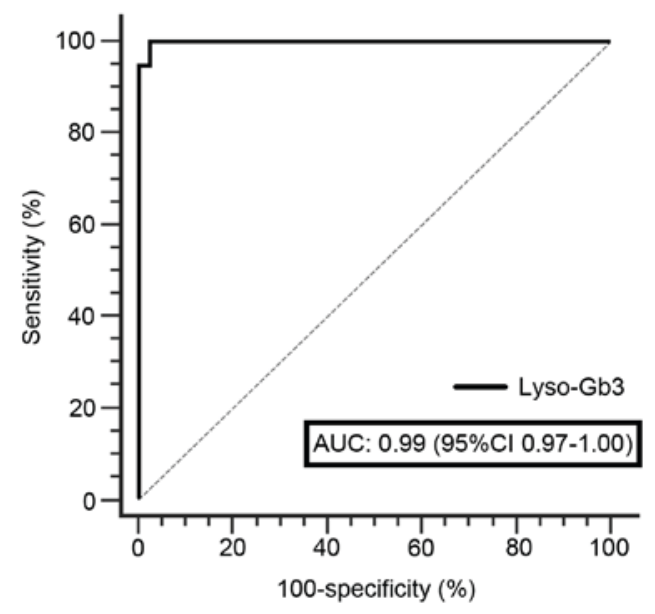

Figure 4. A receiver operating characteristic curve of diagnostic value of lyso-Gb3 between Fabry disease patients and healthy individuals. AUC, area under curve; lyso-Gb3, globotriaosylsphingosine; CI, confidence interval.

levels were observed between healthy males and females. Only trace amounts of it were present in healthy females (median, $0.44 \mathrm{ng} / \mathrm{ml}$; range, $0.25-0.74 \mathrm{ng} / \mathrm{ml}$ ) and healthy males (median, $0.42 \mathrm{ng} / \mathrm{ml}$; range, 0.24-0.81 ng/ml). By contrast, median concentrations in male patients were significantly higher than that in female patients ( $14.50 \mathrm{vs.} 2.79 \mathrm{ng} / \mathrm{ml}$; $\mathrm{P}<0.001$; Table I), as expected. Although male and female patients had significantly higher plasma lyso-Gb3 levels than healthy groups (both $\mathrm{P}<0.001$ ), there was a slight overlap between female patients with FD and healthy individuals (Fig. 3B). The pathological threshold for lyso-Gb3 was set to $0.81 \mathrm{ng} / \mathrm{ml}$ with $100 \%$ specificity and $94.74 \%$ sensitivity, since all samples from healthy controls were clearly below this cut-off value analyzed using a ROC curve (Fig. 4).

Comparison of clinical value between two diagnostic biomarkers. To determine the usefulness of plasma lyso-Gb3

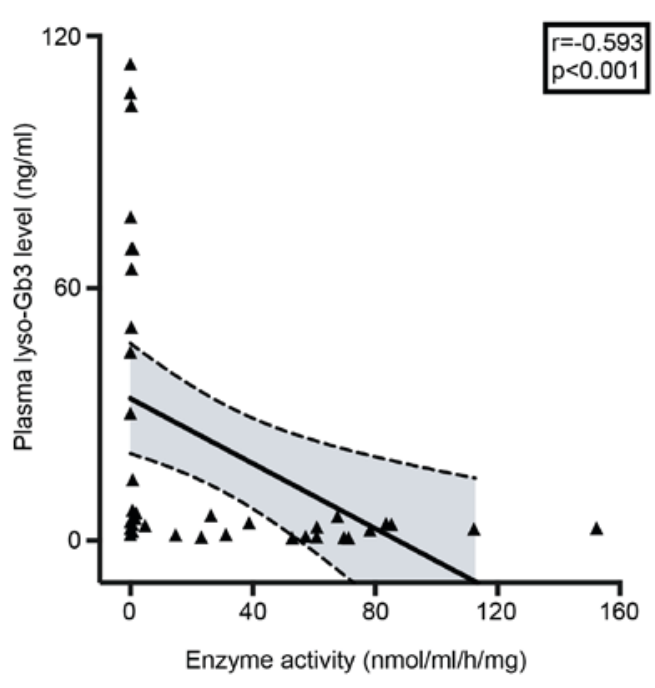

Figure 5. The linear correlation between enzyme activity and lyso-Gb3 in FD patients. Lyso-Gb3, globotriaosylsphingosine.

measurement in confirming FD diagnosis, enzyme activity was measured in all plasma specimens. Linear correlation analysis of all patients revealed a weak-negative correlation between lyso-Gb3 levels and enzyme activity ( $\mathrm{r}=-0.593$; $\mathrm{P}<0.001$; Fig. 5). In males, FD diagnosis could be confirmed by measuring plasma lyso-Gb3 concentration or enzyme activity, as males with FD exhibit markedly higher plasma lyso-Gb3 concentrations and markedly decreased enzyme activity (25). The results of the current study indicated that the sensitivities of two diagnostic indicators were $100 \%$ in males. However, the sensitivity of enzyme activity was markedly lower than plasma lyso-Gb3 (23.5 vs. 82.4\%) in females with FD (Table III).

Due to incomplete results from examinations, 15 patients lacking MSSI scores were excluded. In male patients, plasma lyso-Gb3 and enzyme activity were correlated with MSSI ( $\mathrm{P}<0.01$; Fig. 6A and B). Lyso-Gb3 was observed to be more strongly correlated with MSSI than enzyme activity $(r=0.711$ vs. $r=-0.687$; Fig. 6). However, the analysis of correlation between lyso-Gb3 or enzyme activity and MSSI showed no significant difference in female patients (Fig. 6C and D).

\section{Discussion}

To the best of our knowledge, the current study is the first to measure plasma lyso-Gb3 levels in Chinese patients with FD. A robust LC-MS/MS assay with a short instrument run-time and easy sample handling was developed and used. Instead of choosing the 95th percentile calculation of the normal range $(0.66 \mathrm{ng} / \mathrm{ml})$ as the diagnostic value, a cut-off value of $0.81 \mathrm{ng} / \mathrm{ml}$ was determined by ROC curve analysis. This value, with $94.74 \%$ sensitivity and $100 \%$ specificity, was close to $0.9 \mathrm{ng} / \mathrm{ml}$, the pathological threshold set by Lukas et al (25). In the current study, lyso-Gb3 levels in the majority of patients with FD were above the pathological level of $0.81 \mathrm{ng} / \mathrm{ml}$. In fact, only $17.65 \%$ heterozygote females (3/17) exhibited weakly detectable lyso-Gb3 signals below the cut-off limit. The diagnostic sensitivity of enzyme activity was the same as lyso-Gb3 levels in male patients. However, in female patients, it was remarkably lower than plasma lyso-Gb3 levels. These results indicate 

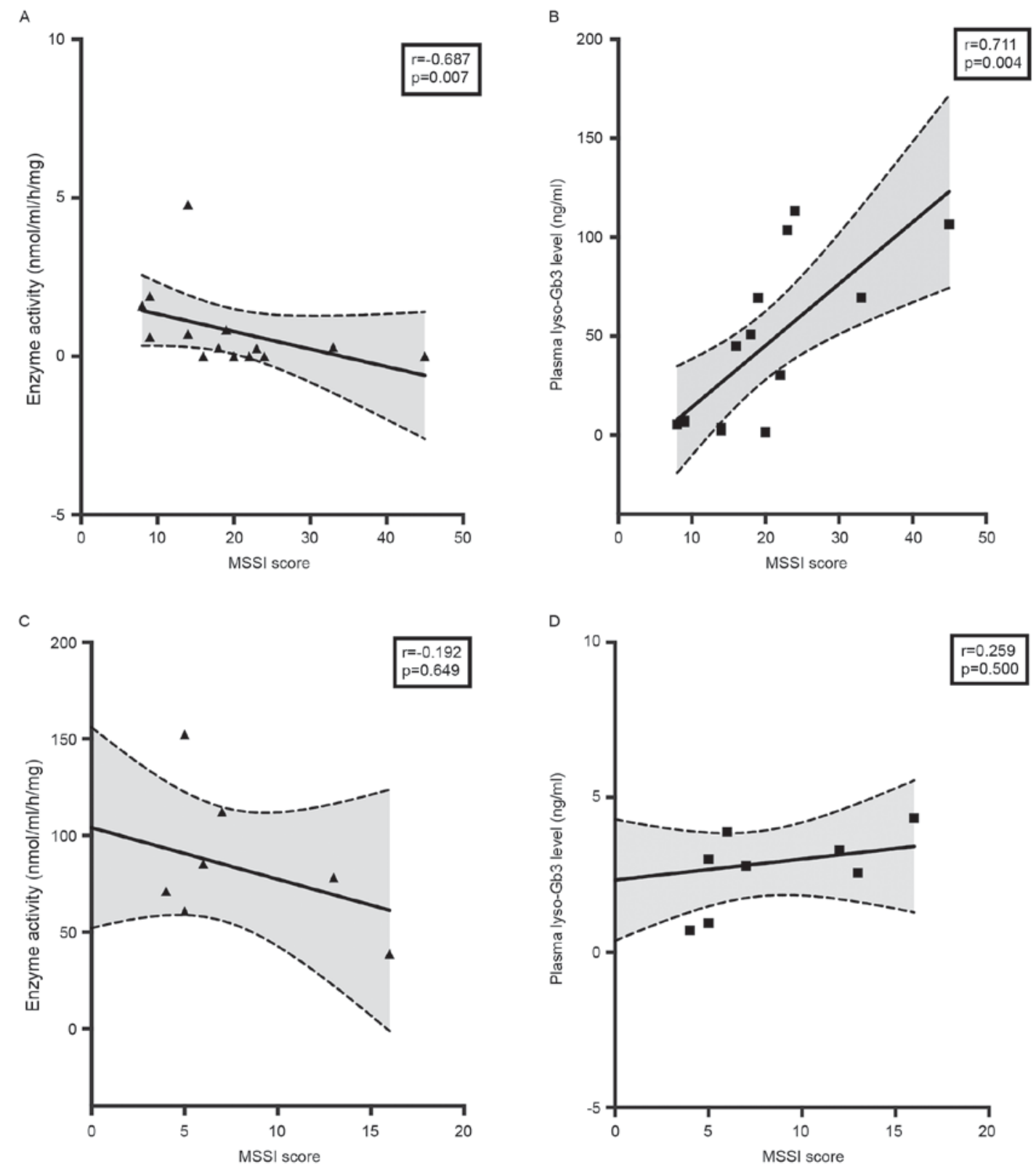

Figure 6. Correlation of MSSI with (A and C) enzyme activity and (B and D) lyso-Gb3 were separately analyzed according to sex. Correlation in males ( $\mathrm{n}=14$, upper panels) and females ( $\mathrm{n}=9$, lower panels) with Fabry disease, are depicted separately. MSSI, mainz severity score index; lyso-Gb3, globotriaosylsphingosine.

that lyso-Gb3 levels are more reliable than enzyme activity at diagnosing patients with FD, particularly in women.

Recently awareness of FD in China has improved; but the incidence of patients receiving delayed diagnoses and misdiagnoses remains high, particularly in women (39). The results of the current study indicate that the incidence of the delayed diagnosis in Chinese patients with FD was 71.1\% (27/38) and the mean delay in diagnosis was 9.61 years (range, 0-47 years). Consequently, the incidence of FD in China is generally underestimated, despite the existence of a large population base (32). Performing a biopsy of affected organs and/or tissues is an effective method of diagnosing FD; however, this is not possible for all patients, for example, in individuals presenting with an isolated stroke (40). Although measuring enzyme activity is the main method of diagnosing FD in males, some female patients exhibit enzyme activity within normal ranges (7). Furthermore, genetic sequencing could not detect all mutations that lead to the development of FD because the patients frequently lacked a family history of the disease. The percentage of undetected mutation was $\sim 10 \%$ (14).

Due to these weaknesses, a novel sensitive diagnostic biomarker for FD is urgently required. In the current study, male patients with FD exhibit markedly higher plasma lyso-Gb3 concentrations and markedly decreased enzyme activity, these results are similar to pervious studies $(21,25)$. The plasma lyso-Gb3 level was confirmed to be a reliable diagnostic indicator of FD in other ethnic groups (21), however it has not been validated in the Chinese population. To the best of our knowledge, this current study is the first time it has been indicated that determining lyso-Gb3 levels and leukocyte enzyme activity are useful biomarkers when diagnosing Chinese male patients with FD. However, the plasma lyso-Gb3 level assay was not $100 \%$ effective, as 
some female patients with FD exhibit nearly normal levels of lyso-Gb3.

The results of a previous study indicated that there is an association between disease severity and the $\alpha$-gal A activity in female patients with FD (32). The observed residual enzyme activity in plasma or blood cells from male patients is a poor predictor of clinical course (32); therefore better clinical indicators are required for male patients. To confirm the clinical application of lyso-Gb3 in the current study, the correlation between MSSI and enzyme activity, as well as between MSSI and lyso-Gb3, were evaluated. The MSSI score was confirmed to be a useful, specific measure for objectively assessing the severity of FD. Due to financial constraints, some patients refused a full set of examinations at diagnosis. The MSSI score could not be assessed in the patients who did not have ultrasonic cardiogram or brain MRI examinations. Due to a lack of comprehensive examination data, 15 patients ( 7 males and 8 females) without MSSI scores were excluded prior to performing the further analyses of MSSI.

In male patients, lyso-Gb3 levels were more strongly correlated with MSSI $(\mathrm{r}=0.711 ; \mathrm{P}=0.004)$ than enzyme activity $(\mathrm{r}=-0.687 ; \mathrm{P}=0.007)$. However, in female patients, lyso-Gb3 and enzyme activity were not correlated with MSSI (Fig. 6C and D). The results were slightly different from those of previous studies $(16,18)$, which identified a strong correlation between lyso-Gb3 and MSSI in females. No correlation was noted between the lyso-Gb3 level and MSSI score in male patients $(19,21)$. This may be due to the fact that the majority of male patients in the aforementioned studies exhibit extremely high lyso-Gb3 levels. Smid et al (41) found the lyso-Gb3 level to be markedly higher in classical FD than atypical FD, especially in male patients. The male patients included in the current study consisted of 10 patients with classical phenotypes and 11 with atypical phenotypes (Table I). As the male patients in the current study did not uniformly exhibit high levels of lyso-Gb3, the correlation analysis between MSSI and lyso-Gb3 concentration is more likely to have statistical significance. Furthermore, the very small number of female patients $(n=9)$ included in the MSSI analysis of the current study may have affected the results.

In conclusion, the results of the current study indicated that plasma lyso-Gb3 levels may be a novel diagnostic biomarker for patients with FD. It is particularly helpful at diagnosing females exhibiting near-normal levels of enzyme activity with FD. Furthermore, the strong correlation between lyso-Gb3 and MSSI in male patient means that lyso-Gb3 levels are more useful than enzyme levels at assessing disease severity in male patients. However, no correlation was identified between lyso-Gb3 and MSSI in female patients. To some extent, the lower number of female patients included in the current study may have resulted in a data bias, which may have affected statistical significance. Therefore, future studies involving a larger cohort of patients with FD are required, to confirm that these results are accurate.

\section{Acknowledgements}

We thank Genzyme Corporation (Cambridge, MA, USA) for their support to this study.

\section{Funding}

This study was supported by grants from the National Basic Research Program of China (973 program) (Grant no. 2012CB517604), the Key Program of Shanghai Science and Technology Commission (Grant no. 08dz1900502), and the National Natural Science Foundation of China (Grant no. 30871001).

\section{Availability of data and materials}

All data generated or analyzed during this study are included in this published article.

\section{Authors' contributions}

NC conceived and designed the study. YO, XP, ZW, HR, YX, $\mathrm{LY}$ and $\mathrm{NC}$ collected the clinical and pathological data patients with Fabry disease. YO and BC performed the analysis of plasma lyso-Gb3. LN and XY performed the enzyme activity assay. XP and XY performed the DNA/RNA sequencing. YO and $\mathrm{BC}$ analyzed the data. YO, BC, XP and $\mathrm{NC}$ wrote and revised the paper. All authors read and approved the final manuscript.

\section{Ethics approval and consent to participate}

The present study was approved by the Ethics Committee of Ruijin Hospital and all participants provided their informed consent prior to participation in the current study. Written informed consent was obtained from the parents or guardians of children enrolled.

\section{Consent for publication}

Not applicable.

\section{Competing interests}

The authors declare that they have no competing interests.

\section{References}

1. Germain DP: Fabry disease. Orphanet J Rare Dis 5: 30, 2010.

2. Askari H, Kaneski CR, Semino-Mora C, Desai P, Ang A, Kleiner DE, Perlee LT, Quezado M, Spollen LE, Wustman BA and Schiffmann R: Cellular and tissue localization of globotriaosylceramide in Fabry disease. Virchows Arch 451: 823-834, 2007.

3. Desnick RJ, Brady R, Barranger J, Collins AJ, Germain DP, Goldman M, Grabowski G, Packman S and Wilcox WR: Fabry disease, an under-recognized multisystemic disorder: Expert recommendations for diagnosis, management, and enzyme replacement therapy. Ann Intern Med 138: 338-346, 2003.

4. Elleder M, Bradová V, Smíd F, Buděsínský M, Harzer K, Kustermann-Kuhn B, Ledvinová J, Bĕlohlávek, Král V and Dorazilová V: Cardiocyte storage and hypertrophy as a sole manifestation of Fabry's disease. Report on a case simulating hypertrophic non-obstructive cardiomyopathy. Virchows Arch A Pathol Anat Histopathol 417: 449-455, 1990.

5. Nakao S, Kodama C, Takenaka T, Tanaka A, Yasumoto Y, Yoshida A, Kanzaki T, Enriquez AL, Eng CM, Tanaka H, et al: Fabry disease: Detection of undiagnosed hemodialysis patients and identification of a 'renal variant' phenotype. Kidney Int 64: 801-807, 2003. 
6. Brady RO, Gal AE, Bradley RM, Martensson E, Warshaw AL and Laster L: Enzymatic defect in Fabry's disease. Ceramidetrihexosidase deficiency. N Engl J Med 276: 1163-1167, 1967.

7. Linthorst GE, Vedder AC, Aerts JM and Hollak CE: Screening for Fabry disease using whole blood spots fails to identify one-third of female carriers. Clin Chim Acta 353: 201-203, 2005.

8. Kitagawa T, Ishige N, Suzuki K, Owada M, Ohashi T, Kobayashi M, Eto Y, Tanaka A, Mills K, Winchester B and Keutzer J: Non-invasive screening method for Fabry disease by measuring globotriaosylceramide in whole urine samples using tandem mass spectrometry. Mol Genet Metab 85: 196-202, 2005

9. Whitfield PD, Calvin J, Hogg S, O'Driscoll E, Halsall D Burling K, Maguire G, Wright N, Cox TM, Meikle PJ and Deegan PB: Monitoring enzyme replacement therapy in Fabry disease-role of urine globotriaosylceramide. J Inherit Metab Dis 28: 21-33, 2005.

10. Rozenfeld PA, De Francesco NP, Borrajo GJ, Ceci R and Fossati CA: An easy and sensitive method for determination of globotriaosylceramide $(\mathrm{Gb} 3)$ from urinary sediment: Utility for Fabry disease diagnosis and treatment monitoring. Clin Chim Acta 403: 194-197, 2009.

11. Young E, Mills K, Morris P, Vellodi A, Lee P, Waldek S and Winchester B: Is globotriaosylceramide a useful biomarker in Fabry disease? Acta Paediatr Suppl 94: 51-54; discussion 37-58, 2005.

12. Blaydon D, Hill J and Winchester B: Fabry disease: 20 novel GLA mutations in 35 families. Hum Mutat 18: 459, 2001.

13. Schäfer E, Baron K, Widmer U, Deegan P, Neumann HP, Sunder-Plassmann G, Johansson JO, Whybra C, Ries M, Pastores GM, et al: Thirty-four novel mutations of the GLA gene in 121 patients with Fabry disease. Hum Mutat 25: 412, 2005.

14. van der Tol L, Smid BE, Poorthuis BJ, Biegstraaten M, Deprez RH, Linthorst GE and Hollak CE: A systematic review on screening for Fabry disease: Prevalence of individuals with genetic variants of unknown significance. J Med Genet 51: 1-9, 2014.

15. Fazekas F, Enzinger C, Schmidt R, Grittner U, Giese AK, Hennerici MG, Huber R, Jungehulsing GJ, Kaps $M$, Kessler C, et al: Brain magnetic resonance imaging findings fail to suspect Fabry disease in young patients with an acute cerebrovascular event. Stroke 46: 1548-1553, 2015

16. van der Tol L, Cassiman D, Houge G, Janssen MC, Lachmann RH, Linthorst GE, Ramaswami U, Sommer C, Tøndel C, West ML, et al: Uncertain diagnosis of fabry disease in patients with neuropathic pain, angiokeratoma or cornea verticillata: Consensus on the approach to diagnosis and follow-up. JIMD Rep 17: 83-90, 2014.

17. van der Tol L, Svarstad E, Ortiz A, Tøndel C, Oliveira JP, Vogt L, Waldek S, Hughes DA, Lachmann RH, Terryn W, et al: Chronic kidney disease and an uncertain diagnosis of Fabry disease: Approach to a correct diagnosis. Mol Genet Metab 114: 242-247, 2015.

18. Smid BE, van der Tol L, Cecchi F, Elliott PM, Hughes DA, Linthorst GE, Timmermans J, Weidemann F, West ML, Biegstraaten M, et al: Uncertain diagnosis of Fabry disease: Consensus recommendation on diagnosis in adults with left ventricular hypertrophy and genetic variants of unknown significance. Int J Cardiol 177: 400-408, 2014

19. Aerts JM, Groener JE, Kuiper S, Donker-Koopman WE, Strijland A, Ottenhoff R, van Roomen C, Mirzaian M, Wijburg FA, Linthorst GE, et al: Elevated globotriaosylsphingosine is a hallmark of Fabry disease. Proc Natl Acad Sci USA 105: 2812-2817, 2008

20. Togawa T, Kodama T, Suzuki T, Sugawara K, Tsukimura T, Ohashi T, Ishige N, Suzuki K, Kitagawa T and Sakuraba H: Plasma globotriaosylsphingosine as a biomarker of Fabry disease. Mol Genet Metab 100: 257-261, 2010.

21. Rombach SM, Dekker N, Bouwman MG, Linthorst GE, Zwinderman AH, Wijburg FA, Kuiper S, Vd Bergh Weerman MA, Groener JE, Poorthuis BJ, et al: Plasma globotriaosylsphingosine: Diagnostic value and relation to clinical manifestations of Fabry disease. Biochim Biophys Acta 1802: 741-748, 2010.

22. Krüger R, Tholey A, Jakoby T, Vogelsberger R, Mönnikes R, Rossmann H, Beck M and Lackner KJ: Quantification of the Fabry marker lysoGb3 in human plasma by tandem mass spectrometry. J Chromatogr B Analyt Technol Biomed Life Sci 883-884: 128-135, 2012.
23. Gold H, Mirzaian M, Dekker N, Joao Ferraz M, Lugtenburg J, Codée JD, van der Marel GA, Overkleeft HS, Linthorst GE, Groener JE, et al: Quantification of globotriaosylsphingosine in plasma and urine of fabry patients by stable isotope ultraperformance liquid chromatography-tandem mass spectrometry. Clin Chem 59: 547-556, 2013.

24. Boutin M, Gagnon R, Lavoie $P$ and Auray-Blais C: LC-MS/MS analysis of plasma lyso-Gb3 in Fabry disease. Clin Chim Acta 414: 273-280, 2012.

25. Lukas J, Giese AK, Markoff A, Grittner U, Kolodny E, Mascher H, Lackner KJ, Meyer W, Wree P, Saviouk V and Rolfs A: Functional characterisation of alpha-galactosidase a mutations as a basis for a new classification system in fabry disease. PLoS Genet 9: e1003632, 2013.

26. Lavoie P, Boutin M and Auray-Blais C: Multiplex analysis of novel urinary lyso-Gb3-related biomarkers for Fabry disease by tandem mass spectrometry. Anal Chem 85: 1743-1752, 2013.

27. Boutin M and Auray-Blais C: Multiplex tandem mass spectrometry analysis of novel plasma lyso- $\mathrm{Gb}_{3}$-related analogues in Fabry disease. Anal Chem 86: 3476-3483, 2014.

28. Auray-Blais C, Boutin M, Gagnon R, Dupont FO, Lavoie P and Clarke JT: Urinary globotriaosylsphingosine-related biomarkers for Fabry disease targeted by metabolomics. Anal Chem 84: 2745-2753, 2012

29. Dupont FO, Gagnon R, Boutin M and Auray-Blais C: A metabolomic study reveals novel plasma lyso-Gb3 analogs as Fabry disease biomarkers. Curr Med Chem 20: 280-288, 2013.

30. Sessa A, Toson A, Nebuloni M, Pallotti F, Giordano F, Battini G, Maglio A, Meroni M, Calconi G, Bertolone G and Gatti P: Renal ultrastructural findings in Anderson-Fabry disease. J Nephrol 15: 109-112, 2002.

31. Desnick RJ, Allen KY, Desnick SJ, Raman MK, Bernlohr RW and Krivit W: Fabry's disease: Enzymatic diagnosis of hemizygotes and heterozygotes. Alpha-galactosidase activities in plasma, serum, urine, and leukocytes. J Lab Clin Med 81: 157-171, 1973.

32. Pan X, Ouyang Y, Wang Z, Ren H, Shen P, Wang W, Xu Y, Ni L, Yu X, Chen X, et al: Genotype: A crucial but not unique factor affecting the clinical phenotypes in fabry disease. PLoS One 11: e0161330, 2016.

33. Levey AS, Stevens LA, Schmid CH, Zhang YL, Castro AF III, Feldman HI, Kusek JW, Eggers P, Van Lente F, Greene T, et al: A new equation to estimate glomerular filtration rate. Ann Intern Med 150: 604-612, 2009.

34. Schwartz GJ, Haycock GB, Edelmann CM Jr and Spitzer A: A simple estimate of glomerular filtration rate in children derived from body length and plasma creatinine. Pediatrics 58: 259-263, 1976.

35. Whybra C, Kampmann C, Krummenauer F, Ries M, Mengel E, Miebach E, Baehner F, Kim K, Bajbouj M, Schwarting A, et al: The mainz severity score index: A new instrument for quantifying the Anderson-Fabry disease phenotype, and the response of patients to enzyme replacement therapy. Clin Genet 65: 299-307, 2004.

36. Bligh EG and Dyer WJ: A rapid method of total lipid extraction and purification. Can J Biochem Physiol 37: 911-917, 1959.

37. NCCLS: Evaluation of Precision Performance of Quantitative Measurement Methods; Approved Guideline-Second Edition. NCCLS document EP5-A2 (ISBN 1-56238-542-9). NCCLS, Wayne, PA, 2004.

38. NCCLS: Evaluation of the Linearity of Quantitative Measurement Procedures: A Statistical Approach; Approved Guideline. NCCLS document EP6-A (ISBN 1-56238-498-8). NCCLS, Wayne, PA, 2003.

39. Jamboti J and Forrest $\mathrm{CH}$ : Fabry disease; early diagnosis improves prognosis but diagnosis is often delayed. J Nephropathol 6: 130-133, 2017.

40. Kes VB, Cesarik M, Zavoreo I, Butković SS, Kes P, Bašić-Jukić N, Rački S, Jakić M, Delić-Brkljačić D, Jukić Z, et al: Guidelines for diagnosis, therapy and follow up of Anderson-Fabry disease. Acta Med Croatica 68: 223-232, 2014.

41. Smid BE, van der Tol L, Biegstraaten M, Linthorst GE, Hollak CE and Poorthuis BJ: Plasma globotriaosylsphingosine in relation to phenotypes of Fabry disease. J Med Genet 52: 262-268, 2015.

This work is licensed under a Creative Commons Attribution-NonCommercial-NoDerivatives 4.0 International (CC BY-NC-ND 4.0) License. 